\title{
Germanica
}

\section{L'écho du champ de bataille. La Saga de Gösta Berling comme roman impérial}

Das Echo des Schlachtfeldes Gösta Berlings Saga als Roman des Empire

\section{Vivi Edström}

\section{CpenEdition}

\section{Journals}

Édition électronique

URL : http://journals.openedition.org/germanica/1271

DOI : 10.4000/germanica. 1271

ISSN : 2107-0784

Éditeur

Université de Lille

\section{Édition imprimée}

Date de publication : 1 décembre 1998

Pagination : 119-128

ISBN : 0984-2632F

ISSN : 0984-2632

\section{Référence électronique}

Vivi Edström, «L'écho du champ de bataille. La Saga de Gösta Berling comme roman impérial », Germanica [En ligne], 23 | 1998, mis en ligne le 26 janvier 2012, consulté le 06 octobre 2020. URL http://journals.openedition.org/germanica/1271 ; DOI : https://doi.org/10.4000/germanica.1271

Ce document a été généré automatiquement le 6 octobre 2020.

(c) Tous droits réservés 


\title{
L'écho du champ de bataille. La Saga de Gösta Berling comme roman impérial $^{1}$
}

Das Echo des Schlachtfeldes Gösta Berlings Saga als Roman des Empire

\author{
Vivi Edström
}

1 La Saga de Gösta Berling (Gösta Berlings saga),livre par lequel Selma Lagerlöf débuta dans la littérature, parut en 1891. Ce roman rompt avec le réalisme des années 1880, avec son engagement social et son intérêt pour l'époque contemporaine. Les écrivains de la décennie suivante, celle des années 1890 , se tournent vers le passé. C'est dans le Moyen Age, la Renaissance et le Romantisme qu'ils cherchent la matière susceptible de nourrir les émotions et d'inspirer des élans poétiques. La distance prise par rapport au présent se prête plus facilement à l'emphase, à l'exaltation de sentiments, à la nostalgie et au mélodrame.

2 La Saga de Gösta Berlingest un livre de la bravade et de l'hyperbole. L'existence autour du long lac Löven est présentée par Selma Lagerlöf comme riche en aventures et en personnages hauts en couleur. Tout est poussé à l'extrême. Dans le grand domaine Ekeby se déroule «la plus magnifique des fêtes». "Où ailleurs pouvait-on voir des parquets aussi brillants, des hommes aussi courtois, des femmes aussi belles? " Le héros et le Don Juan du roman, le pasteur défroqué Gösta Berling, est présenté comme " un roi parmi les hommes». La jeune Marianne Sinclaire, une des femmes qui lui ont inspiré de l'amour, est décrite comme "la plus belle de toutes les belles femmes", comme « une souveraine ».

3 Tous ces superlatifs conviennent à merveille au rythme intense de l'action. Tout est en mouvement dans ce livre; on est constamment en train de traverser les étendues du lac gelé ou bien de parcourir ses bords en traîneau. Un mouvement qui correspond aux trépidations incessantes de l'âme entre le ravissement et la souffrance.

4 Cette atmosphère exaltée est mise en relation avec une époque bien précise, celle des années 1820, du Romantisme tardif. La houle des guerres révolutionnaires venait alors 
d'atteindre les côtes de la Suède. Nous avons même eu un nouveau monarque venu de l'Europe dynamique: Charles XIV Jean, Bernadotte de son vrai nom et général de l'armée de Napoléon. Le nom de ce roi est étroitement associé à une esthétique : pour les Suédois, le "style Charles Jean" est le synonyme du style Empire. La même sensibilité est dépeinte dans La Saga de Gösta Beding².

5 L'Empire se caractérise par une prédilection pour la symbolique martiale. Les rideaux et les baldaquins de lit, les canapés et les glaces portent des emblèmes guerriers : des pointes de lance, des pistolets croisés, des casques, des aigles et des boulets de canon dorés. Un parfait exemple du style Empire suédois est Rosendal, château de chasse de Charles XIV Jean, qui s'élève à Djurgården à Stockholm. Au Musée National de Stockholm on peut voir un grand portrait du roi, en tenue militaire, portant des bottes, au milieu d'une multitude d'objets de valeur, entouré d'une aura de toute-puissance; dans le fond, se devine un portrait de Napoléon lui-même ${ }^{3}$.

6 Ce «goût pour le pouvoir» est également présent dans La Saga de Gösta Beding, en commençant par le portrait du protagoniste, tel qu'on le trouve dans l'introduction. Gösta Beding est décrit à la fois comme un génie « aux yeux profonds de poète » et comme une statue de guerrier antique. "Sa tête eût-elle été coiffée d'un heaume et son torse revêtu d'une armure, on aurait pu le sculpter en marbre et donner à cette image le nom du plus beau des Athéniens. » Lorsque, du haut de sa chaire, il regarde les paroissiens, son attitude rappelle celle d'un chef militaire, et les gens se sentent " curieusement subjugués » en présence de leur pasteur.

7 Mme la commandante, patronne d'Ekeby, « la dame la plus puissante du Värmland ${ }^{4}$ » est, à l'instar de Charles Jean, une usurpatrice. S'étant emparée du pouvoir par sa propre force, elle tient sous sa houlette même les notables locaux. «Si je lève un doigt, le gouverneur accourt, si j'en lève deux, l'évêque en fait de même, et si j'en lève trois, alors aussi bien le chapitre que les conseillers, ainsi que tous les propriétaires terriens du Värmland se mettent à danser la polonaise sur la place de Karlstad », confie-t-elle à Gösta Berling. Derrière cette raillerie lourde de menaces se devine le mépris de cette femme forte à l'égard des messieurs poltrons. Ceux-là même qui n'oseront pas lever le petit doigt pour la défendre le jour où son trône vacillera.

8 Nous faisons la connaissance de la commandante, revêtue « de la splendeur et de la magnificence» de son rang dans le chapitre intitulé «Le Repas de Noël». Elle est entourée de notables; «sa robe de soie froufroute, ses bras nus sont chargés d'or, des perles rafraîchissent sa poitrine blanche. » Elle "se rengorge » d'être placée entre le doyen de Bro et le comte de Borg - et son triomphe a quelque chose de cette " hybris » qui, depuis l'antiquité, amène immanquablement la chute des fortes personnalités, au moment même où elles se trouvent à l'apogée du succès. Une seule erreur suffit pour que le pouvoir s'écroule.

9 L'erreur de la commandante consiste à avoir manqué d'égards envers la bande de « chevaliers» qui vivent comme des piques-assiettes chez elle à Ekeby. Ces derniers enragent de se voir traités avec dédain lors du magnifique dîner à Ekeby. A table, ils ont été placés trop loin du centre du pouvoir et de la splendeur. «Ah, pourquoi a-t-elle placé les chevaliers à une table obscure, dans un coin!» s'écrit le narrateur avec angoisse. Cette offense est à l'origine de leur révolte contre celle qui a été leur bienfaitrice et leur souveraine. Une révolte qui causera sa chute et la perte de son pouvoir. 
10 L'excès d'orgueil éprouvé lors d'une représentation officielle précède également la chute du pasteur le jour où, au début des années 1820, il gravit enfin les marches d'une chaire dans « une paroisse reculée à l'ouest du Värmland ». La datation de l'événement dans le chapitre d'introduction est assez précise. Ensuite, Gösta Beding passe sept ans chez la commandante à Ekeby, dans l'aile du manoir, qui abrite les « chevaliers », avant de s'emparer, avec ses onze camarades, des sept domaines qui appartiennent à la dame. Certes, nous avons affaire à un conte - le nombre magique, "sept", l'indique clairement -, mais ce conte a ses racines dans l'histoire.

11 Au début, le lien entre les événements décrits et la réalité historique avait été marqué encore plus nettement. Dans une des ébauches du chapitre «La Nuit de Noël », on peut lire que "l'année des chevaliers" débuta à Noël, en $1830^{5}$. Selma Lagerlöf dut certainement comprendre que cette année exceptionnelle et miraculeuse n'a rien à voir avec la chronologie normale. Dans la version publiée, l'auteur se contente de dire dans « Le pays ", qui est un chapitre d'introduction et de mise en place - que l'action se déroule dans les années 1820 .

12 La Saga de Gösta Bedingne correspond pas exactement à ce qu'il est convenu d'appeler «roman historique». Cependant, la problématique historique y est parfaitement perceptible. En outre, ici comme dans plusieurs autres romans de Lagerlöf, l'air du temps joue un rôle primordial.

13 A l'instar de Thomas Carlyle dans La Révolution française,livre dont elle s'était inspirée pour peindre le panorama social dans La Saga de Gösta Beding, Selma Lagerlöf présente la succession d'événements comme une "culbute générale ". Un historien de la littérature, Gunnar Brandell, a remarqué que ce bouleversement n'a pas d'analogies dans la réalité historique des années 1820 . Durant la décennie décrite dans le livre, la situation économique de la région est restée parfaitement stable ${ }^{7}$.

La période marquée par les convulsions, lors desquelles l'agriculture de la région périclita et le Värmland devint une province pauvre, se situe en réalité beaucoup plus tard, dans la seconde moitié des années 1870, pendant l'enfance et l'adolescence de Selma Lagerlöf (née en 1858). Cette crise est décrite dans la seconde partie de l'autobiographie, Mémoires d'un enfant / Ett barns memoarer(1930), où elle est incorporée au récit de l'enfance de l'héroïne. Dans le chapitre "Tremblement de terre ", l'auteur évoque le sentiment d'angoisse devant la ruine qui menace: «Je ferme les yeux et je vois la terre trembler: les grands manoirs s'effondrent, l'un après l'autre. Voici Rottneros qui tombe, et après lui Skarped; Öjervik n'est plus qu'un amas de ruines, de même que Stöparfors, Lövstafors, Gylleby, Helgeby. Herrestad est déjà tombé, et Gårdsjö s'ébranle. »

C'est ce bouleversement qui se reflète dans La Saga de Gösta Berling.Le sentiment de l'existence qui s'écroule, éprouvé quand Mårbacka ${ }^{8}$, lui aussi, fut touché par le tremblement de terre, est à l'origine de la montée de l'inquiétude qui porte cette œuvre de jeunesse de Selma Lagerlöf. Une expérience mouvementée qu'elle relègue dans le passé et qu'elle associe aux années 1820.

16 Pourquoi le fait-elle? La raison principale est certainement dans le fait que l'époque choisie, celle du Romantisme tardif, offre une possibilité de dépeindre les choses en grand, qu'elle permet une intensification et une condensation émotionnelles dont il a été question au début cet article. Considérez, par exemple, l'épisode du voyage en traîneau de Gösta Berling et d'Anna Stjärnhök, dans le chapitre « Gösta Berling, poète », 
où d'un geste significatif, conformément à l'esprit de l'époque, le protagoniste jette à la meute de loups Corinnede Madame de Staël, afin de distraire les fauves! En rattachant sa narration aux années 1820 , Selma Lagerlöf réussit à créer une aura de splendeur et de puissance autour de ses personnages.

Le parfum héroïque émane avant tout des "chevaliers». Dans le chapitre "La cachucha", ils sont décrits d'une façon allégorique comme des destriers que la trompette appelle sans cesse à de nouveaux exploits. Ensemble, ils forment une confrérie de douze héros ou de douze dieux que l'on rencontre fréquemment dans la littérature et la mythologie. Mais La Saga de Gösta Berlingse distingue par le fait que l'éclat martial y est fortement estompé. Au cours de la narration, les « chevaliers » sont décrits comme une bande de militaires à la retraite: le colonel Beerencreutz, le commandant Fuchs, "redoutable chasseur d'ours », le capitaine Bergh, le lieutenant Aquilon, l'enseigne von Ömeclou et ainsi de suite jusqu'au petit tambour Ruster - le grade du personnage est toujours indiqué avec précision.

18 La coterie comprend également "cousin Christopher ", chef militaire énigmatique, ayant servi dans l'armée de Napoléon, avant d'avoir trouvé refuge parmi les " chevaliers » à Ekeby. Son emblème est l'aigle, symbole évident du pouvoir impérial et de l'Empire. Lorsqu'il s'apprête à demander la main de Marthe, comtesse de Borg, il revêt son uniforme militaire brodé d'or. Et plus tard, en voyant son rêve de beauté s'évanouir, il se transforme en un pauvre oiseau mortellement blessé.

Le dynamisme martial, caractéristique de l'esprit de l'époque, se manifeste à plusieurs niveaux. Chez le personnage de Marthe Dohna, la soif de vie effrénée tourne au sadisme. Cette coquette admirée, qui se trouve toujours au centre des événements, dont les plaisanteries furent à l'origine des hostilités et qui avait joué à l'écarté avec les trônes comme enjeu, représente dans le roman le courant néfaste d'autodestruction. Son destin reçoit son atroce épitaphe dans le chapitre «La sorcière du Dovre » où les pies, ces envoyées de la Nemesis, se ruent sur elle.

20 Les vents provenant des champs de bataille européens se font sentir dans l'univers riche en aventures du Värmland, à travers les personnages du cousin Christopher et de Marthe Dohna. Mais Marianne Sinclaire n'appartient-elle pas, elle aussi, à la bouillonnante « jet society » continentale, au sein de laquelle elle évolue, ne daignant que rarement honorer de sa présence le Värmland, ce "pays d'ours »? Grâce à elle, la thématique héroïco-militaire envahit le domaine des sentiments. Marianne fait partie de ces « belles » dont « l'éclat du nom » rehausse la « gloire du Värmland ». « Les récits de ses victoires remplissaient le pays. On dénombrait les couronnes comtales prêtes à se poser sur sa tête, les millions qui avaient été jetés à ses pieds, les épées de soldats et les couronnes de poètes dont la magnificence l'avait attirée ».

21 L'attrait qu'exerce le pouvoir et le vide qu'il recèle se reflètent dans La Saga de Gösta Bedingde diverses façons. Lorsque les « chevaliers " prennent possession des propriétés de la commandante, ils deviennent les rois de la contrée. Ils emploient leur pouvoir à dilapider le capital et à ruiner la confiance qui va avec, tout en brûlant - à un niveau héroïco-parodique - du désir ardent de " défendre l'éclat et l'honneur d'Ekeby ».

22 Le domaine d'Ekeby, foyer de la gaieté, fait davantage penser à une propriété skanienne ou à celle du Sörmland ${ }^{9}$ qu'à un domaine värmlandais. En fait, on ne nous dit pas grandchose sur l'aspect extérieur de l'imposant manoir (et on ne peut que s'étonner que le critique Olof Lagercrantz ait qualifié le roman d'un magasin de meubles!). Dans le chapitre intitulé "Les vieux véhicules", où la commandante s'introduit en cachette 
dans son ancienne maison, il est question de grands et larges sofas, de « dalles fraîches, soutenues par des griffons dorés, sur lesquelles reposaient les glaces ornées d'un trio de déesses dansantes!»-un style Empire typique qui adorait se voir refléter dans les hautes glaces rehaussées de dorure et de marbre.

Dans le chapitre "Madame Musica", on retrouve les "griffons dorés des tables en marbre blanc ». D'autres détails renvoient également à la représentation traditionnelle du style Empire : «Les déesses blanches dansaient sur la surface sombre au-dessus des miroirs ». A la même occasion sont mentionnées les chaises tapissées de tissus fleuris, caractéristiques du style en question.

Le propre de Selma Lagerlöf est de choisir un petit nombre de détails raffinés et de les placer là où il faut, pour réussir à recréer le parfum de l'époque d'une façon parfaitement naturelle - tandis que son amie Sophie Elkan ${ }^{10}$ ruinait souvent l'effet de ses romans historiques en surchargeant le décor et en insistant trop lourdement sur la couleur de l'époque.

La composante historique dans La Saga de Gösta Berlingparticipe de la création de l'atmosphère spécifique du roman. Dans le chapitre «Madame Musica », les chevaliers forment un orchestre et jouent du Haydn «avec lenteur et avec une douceur envoûtante ». Mais l'illusion du doux classicisme est anéantie par l'irruption d'un élément irrationnel. L'épisode central du chapitre est celui où le "chevalier " égaré, Löwenborg (faut-il y voir une allusion à Swedenborg ?) joue du Beethoven sur le clavier dessiné sur une table en bois. Aucun son ne se fait entendre, tout se joue dans la tête du malheureux personnage. Mais l'émotion est là, les sentiments inhibés font leur irruption, ici comme à maints autres endroits du roman. Du reste, Beethoven est précisément lecompositeur de l'Empire, celui qui a exprimé la soif des exploits et de la beauté, l'auteur de l'«élan terrible $»^{11}$, de la musique pour héros, telle La Bataille de Vittoriaou la Symphonie héroïque, dédiée à Napoléon.

La musique de Löwensborg est un rêve qui ne supporte pas le contact avec la réalité. Il en va de même pour l'illusion héroïque tout court. Sa corde est si tendue que, inévitablement, elle se rompt. La chute et la déchéance guettent l'être humain. Ce thème est introduit dès le premier chapitre, dans lequel le pasteur se voit déchu de sa fonction de représentant du Très-Haut. Il déchoit également du point de vue social. Le fonctionnaire se transforme en un mendiant voleur. Lorsqu'il renonce au suicide et qu'il trouve refuge à Ekeby comme chevalier servant de la commandante, il devient un exclu, vomi par Dieu et rejeté par les hommes.

La chute de la commandante est encore plus pathétique. Lorsque les rênes du pouvoir lui échappent et qu'au terme de sa déchéance elle devient une pénitente avec « sac de mendiant et bâton de pèlerin ", cet événement est lourd de conséquences pour toute la contrée. "Car qui restera debout si moi je suis tombée ", s'écrie-t- elle dans la scène où elle quitte Ekeby dans le chapitre «Le repas de Noël ». Elle et Gösta Berling sont les prototypes de tous ceux qui, par la suite, seront attirés par la «chasse effrénée aux aventures » menaçant de détruire l'ordre social établi.

28 La chute et l'humiliation constituent le prix à payer pour les instants de triomphe, le prix de la splendeur et du pouvoir. Marianne Sinclaire ne vaut guère mieux qu'une " esclave châtiée » lorsque, dans le chapitre "Le Bal à Ekeby ", elle se retrouve dehors, dans la nuit glaciale, après que son père, ivre et fou de rage, lui ferme la porte de la demeure. La créature adulée, courtisée par des aristocrates et des militaires couverts de gloire, elle, qui avait été jadis la prunelle des yeux de son père, se voit condamnée par 
lui comme une femme déchue pour avoir embrassé Gösta Berling, son chevalier servant. La scène qui montre Marianne vêtue de sa robe de velours noir, étendue sur la neige, constitue l'apogée théâtral du roman. Elle présente d'une façon condensée l'idée de la vanité fondamentale du pouvoir et de la splendeur (de l'Empire!) qui sont méthodiquement discrédités au cours de la narration.

29 A la fin, le caractère autosuffisant du gai royaume est aboli, lui aussi, et son centre Ekeby - périt dans les flammes. Gösta Berling renonce à la posture héroïque, à la richesse et à la gloire. Il n'a pas la moindre envie de devenir un propriétaire terrien ${ }^{12}$, emploi qui, dans le vocabulaire social de Selma Lagerlöf, désigne une place très enviable sur l'échelle hiérarchique.

Non, son projet est bien plus modeste : vivre dans une cabane de paysan et apprendre aux gens à cultiver les pommes et à chanter des chansons. Cela modifie considérablement l'atmosphère du roman. La paix, la simplicité, le souci démocratique remplacent désormais la "chasse effrénée", ce mouvement révolutionnaire et destructeur qui se manifestait à travers la multitude des symboles de l'Empire.

Si, dans le premier chapitre du roman, Gösta Berling fait surtout penser à quelqu'un comme Tegnér ${ }^{13}$ - l'incarnation même du poète de l'Empire - dans le chapitre qui clôt la narration, il fait figure d'un Atterbom ${ }^{14}$ ou d'un Almqvist ${ }^{15}$ de la première période. Le Romantisme a vaincu l'Empire.

\section{NOTES}

1. Traduit par Elena Balzamo.

2. Dans ma thèse Livets stigar. Tiden, handlingen och livskänslan $i$ Gösta Berlings saga / Sentiers de la vie. Le temps, l'action et l'atmosphère dans La Saga de Gösta Berling (1960), je fais un examen détaillé de diverses composantes temporelles du roman. Dans le présent article, j'essaie d'esquisser une approche différente, en accentuant les liens avec l'empire.

3. L'empire constitue l'objet de plusieurs essais dans Empiren i Sverige. Bildkonst, konsthantverk och inredningar 1800-1844 / L'Empire en Suède. Arts plastiques, artisanat et décoration 1800-1844, catalogue de l'exposition au Musée National du 15 octobre 1991 au 29 mars 1992.

4. Province suédoise, région natale de Selma Lagerlöf. N.d.T.

5. Cf. Livets stigar, p. 1, Note 3.

6. En français dans le texte. N.d.T.

7. Gunnar Brandeli, Revolt $i$ dikt och andra studier / La révolte dans la littérature et autres essais, Stockholm 1977, p. 38.

8. Maison natale de Selma Lagerlöf. N.d.T.

9. La Scanie et le Sörmland (Södermanland) sont les provinces suédoises caractérisées par un climat plus clément et une nature moins austère que le Värmland. N.d.T.

10. (1853-1921), amie de S. Lagerlöf, auteur, entre autres, de romans historiques. N.d.T.

11. En français dans le texte. N.d.T.

12. Littéralement "maître de forges" (brukspatron), terme qui fait davantage allusion à la position clé de la personne dans l'économie de sa région qu'elle n'indique son emploi véritable. N.d.T. 
13. Esaias Tegnér (1782-1846), célèbre poète suédois. N.d.T.

14. Per Atterbom (1790-1855), poète romantique et historien de la littérature. N.d.T.

15. Carl Johan Love Almqvist (1793-1866), écrivain suédois, romantique à ses débuts, réaliste par la suite. N.d.T.

\section{RÉSUMÉS}

L'objectif de l'article consiste à analyser le roman La Saga de Gösta Berling à la lumière de l'esthétique de l'Empire. A cette fin, l'auteur s'attache à relever les attributs du style qui opèrent à différents niveaux de la narration, avant de procéder à leur classement et à leur analyse. On se trouve ainsi en présence d'un télescopage de plusieurs époques : il apparaît que, dans son roman, Selma Lagerlöf recourt à la signalétique de la période du Romantisme tardif pour dépeindre les processus qui, en réalité, sont postérieurs de plusieurs décennies (et qui en outre relèvent de son expérience personnelle). Cette technique lui permet à la fois d'élucider certains mécanismes à l'œuvre durant la période mise à contribution et, en même temps, d'atteindre l'effet esthétique voulu, en utilisant dans sa narration divers procédés de l'écriture romantique tout en prenant ses distances par rapport à ces derniers.

Das Ziel dieses Artikels besteht darin, den Roman Gösta Berlings Saga unter dem Aspekt der Ästhetik des Empire zu analysieren. Deswegen beschäftigt sich der Autor damit, auf die stilistischen Merkmale einzugehen, die auf verschiedenen Ebenen der Erzählung wirksam werden, bevor er sie bewertet und analysiert. So steht man einem Aufeinandertreffen von verschiedenen Epochen gegenüber: Es wird sichtbar, daß Selma Lagerlöf in ihrem Roman sich der Stilmerkmale der Spätromantik bedient, um Ereignisse zu schildern, die in Wirklichkeit mehrere Jahrzehnte später stattfinden (und die außerdem aus ihrem persönlichen Erfahrungsbereich stammen). Diese Technik erlaubt es ihr, am Werk gewisse Mechanismen aus der herangezogenen Epoche zu erhellen und gleichzeitig die gewollte ästhetische Wirkung zu erreichen, indem sie in ihrer Erzählung verschiedene romantische Schreibtechniken verwendet und sich gleichzeitig von ihnen distanziert. 